\title{
Underwater Passive Location Technology Using Dummy Time-Reversal Mirror
}

\author{
Xueli Sheng, Xizhong Bao, Junying Hui, and Guolong Liang \\ College of Underwater Acoustic Engineering, Harbin Engineering University, \\ HLJ, P.R. China \\ Shengxueli@yahoo.com.cn
}

\begin{abstract}
Time reversal (TR) refocuses the incident acoustic field back to the origin of a probe signal regardless of the complexity of the medium. However, the operation of a TR requires a cooperative probe source. This is impossible in passive work mode. In this paper, a dummy time-reversal mirror (DTRM) method is discussed to introduce the concept of time-reversal into underwater passive location. Several ocean acoustics experiments demonstrating the implementation of DTRM have been conducted. The proposed method is useful while at long source-receive ranges, or while object is moving.
\end{abstract}

Keywords: Dummy Time-reversal mirror, Passive location, adaptive refouse.

\section{Introduction}

Ideal channel can transform information invariably but underwater acoustic channel cannot because it's multipath character. The focusing of acoustic waves in inhomogeneous media is a common and difficult problem.

The concept of an acoustic time reversal mirror was introduced by Fink in 1989 as an extension of the concept of optical phase-conjugate mirror [1]. In 1991, Jackson and Dowling presented the idea of adapting the techniques of optical phase-conjugate mirrors to problems in underwater acoustics [2]. An experiment conducted by $\mathrm{Ku}-$ perman in the Mediterranean Sea in April 1996 demonstrated that a time-reversal mirror can be implemented to spatially and temporally refocus an incident acoustic field back to its origin [3]. Over the last twenty years, acoustic time-reversal mirror have been demonstrated in the ocean and now the important application of time-reversal is in underwater communications, water tunnel acoustic measurements, reverberation nulling, etc[4],[5].

The use of time-reversal in the field of underwater passive location is a relatively new field. Generally, the typical time-reversal experiment needs a source to emit signal and a source array to re-emit time reversed signal. It is hard to be satisfied while locating a passive source. In this study, passive location technology based on an acoustic dummy time-reversal mirror (DTRM) is investigated. Instead of using a source array to re-emit reversed signal, model of Coherent Multipath Channels is established and the method makes use of established channels instead of actual ocean medium. Time reversed signal is convoluted with established channels by computer, 
but not retransmitted to ocean. In the next section, TRM theory is reviewed. In Sec. 2, the proposed DTRM method is described and simulative results are given. In Sec. 3, experimental geometry and experiment results are described. Conclusions are drawn in Sec. 4.

\section{Background Theory}

The theory of time-reversal has already been presented [2],[6],[7]. Here we briefly review salient issues using the basic geometry of Kuperman's TRM experiment (shown schematically in Fig.1.

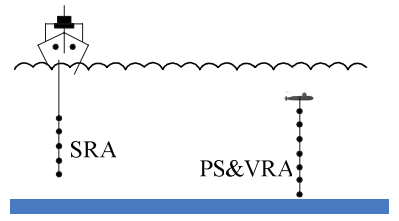

Fig. 1. Kuperman's experimental setup of TRM

A probe source (PS) indicated by one of the rectangles on the vertical receive array (VRA), sends out a pulse that is received at the source-receive array (SRA). The dispersed signal with all its multipath structure is time reversed and retransmitted by the SRA. The resulting signal multi-path structure collapses to a spatial and temporal focus at the original PS position that is co-located in range with the VRA.

Underwater acoustic channel can be regarded as a coherence and multipath channel while its spatial and temporal randomization is neglected [8]. We demonstrate the temporal and spatial adaptive focusing character of TRM from the point of view of ray theory.

Define $R_{i}(\omega)$ as the Fourier transform (FT) of $r_{i}(t)$, the signal received by the $i$ th SRA receiver element. $R_{i}(\omega)$ can be described as the product of $S(\omega)$, the FT of the signal emitted by PS, and $H_{i}(\omega)$, channel's transform function from PS to the $i$ th receiver element of the SRA

$$
R_{i}(\omega)=H_{i}(\omega) \cdot S(\omega)
$$

Define $X_{i}(\omega)$ as the complex conjugate of $R_{i}(\omega)$, which is equal to time reverses $r_{i}(t)$ at time-domain.

$$
X_{i}(\omega)=R_{i}^{*}(\omega)=H_{i}^{*}(\omega) \cdot S^{*}(\omega)
$$

Both side of Eq. (2) multiplied by $H(\omega)$, channel's transform function from the $i$ th SRA element to somewhere in the ocean. The solution is given by

$$
Z(\omega)=H(\omega) \cdot X_{i}(\omega)=H(\omega) \cdot H_{i}^{*}(\omega) \cdot S^{*}(\omega) .
$$


$Z(\omega)$ can be regarded as the output of the TRM. Eq. (3) can be used to show that the TRM produces focusing in time as well as in space. Examine the TRM output at the focus point, that is, takes $H(\omega)=H_{i}(\omega)$. " $H_{i}(\omega) \cdot H_{i}^{*}(\omega)$ " in Eq. (3) is a real, even and positive function where the maximum of correlation can be got when IFT executed at $\mathrm{t}=0$. This operation is matched filtering. The sum over SRA elements is a form of spatial matched-field processor.

The principle demonstrated upwards is the basic of acoustic time-reversal mirror in a range-independent waveguide.

\section{Dummy Time-Reversal Mirror (DTRM)}

\subsection{Theory on Passive Location Using a Dummy Time-Reversal Mirror}

As described above, TRM works as a form of matched filtering.

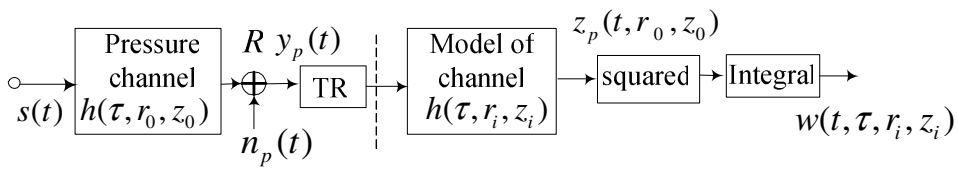

Fig. 2. Principle block diagram of DTRM

Principle block diagram of DTRM is shown in Fig. 2. Here, $s(t)$ is the noise radiated by passive point source located at $a_{0}\left(r_{0}, z_{0}\right) \cdot h\left(\tau, r_{0}, z_{0}\right)$ is channel's transform function from passive source to receiver $R$ and $y_{p}(t)$ is receiver's output that ambient noise be taken into account. A mathematical model of coherent multipath channel from receiver to every interested point $a_{i}\left(r_{i}, z_{i}\right)$ (include $\left.a_{0}\right)$ in the ocean, which marked as $h\left(\tau, r_{i}, z_{i}\right)$, can be established by using sound speed, depth of sea area and sea bottom character measured practically. The received signal $y_{p}(t)$ is time reversed and 'retransmitted' by computer and the resulting signal $z_{p}\left(t, r_{i}, z_{i}\right)$ is an equivalence of the TRM reslut at the position $a_{i}\left(r_{i}, z_{i}\right)$. In this course, $y_{p}(t)$ dose not retransmitted to ocean factually but convoluted with channel model $h\left(\tau, r_{i}, z_{i}\right)$ by computer. $z_{p}\left(t, r_{i}, z_{i}\right)$ can be regarded as IFT of $Z(\omega)$ shown in Eq. 3, which multipath structure collapses at the original passive point source location $a_{0}\left(r_{0}, z_{0}\right)$, that is mean the energy $w\left(t, \tau, r_{0}, z_{0}\right)$ of $z_{p}\left(t, r_{0}, z_{0}\right)$ is maximum of all $w\left(t, \tau, r_{i}, z_{i}\right)$. This is the concept of passive location by DTRM.

\subsection{Model of Coherent Multipath Channel}

In this paper, a mathematical model of coherent multipath channel is established by using the theory of ray acoustic and the method of eigenrays to simplify the physical 
of underwater sound channel. Multipath channel's system function is determined by eigenrays which are sound rays reaching the receive point. The sea surface and sea bottom has a profound effect on propagation in most applications of underwater sound where source or receiver lie at shallow depth. Above all, establishing coherent multipath channel model consists of two cruces. First, search all the important eigenrays quickly and correctly. Secondly, estimate acoustic parameters of sea surface and sea bottom precision accurately ${ }^{[9]}$.

The method of establishing a model of coherent multipath channel based on the theory of ray acoustic and eigenrays is described in [9].

A criterion for the roughness or smoothness of a surface is given by the Rayleigh parameter $^{[10]}$ and the average coefficient of sea surface is related to frequency by

$$
V_{s}=1-0.45 \cdot\left(f \cdot H_{\text {avg }}\right)^{3 / 2} \cdot \cos \theta_{0} .
$$

where $f$ is the frequency in kilohertz, and $H_{\text {avg }}$ is wave's average height in meter.

Acoustic parameters of sea bottom is estimated by using the three-parameters model $^{[11]}$

$$
-\ln \left|V_{b}(\theta)\right|=\left\{\begin{array}{ll}
Q \cdot \theta & 0<\theta<\theta^{*} \\
-\ln \left|V_{b 0}\right|=\text { const } & \theta^{*}<\theta<\frac{\pi}{2}
\end{array} .\right.
$$

A critical angle $\theta^{*}$ exists such that complete reflection occurs at grazing angles $\theta$ less than critical. $Q$ is the slope of sea bottom reflection at grazing angles less than $\theta^{*}$ and $V_{b 0}$ is sea bottom reflection at grazing angles more than $\theta^{*}$. Description of the three-parameters model is shown in Fig. 3.

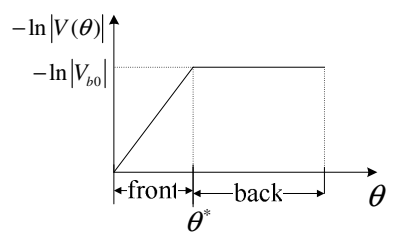

Fig. 3. Three-parameters model

The amplitude $A$, time delay $\tau$, and grazing angle $\theta$ of eigenrays reaching a given point in the sea can be got from model of coherent multipath channel and the pressure channel from this point to source can be described as

$$
h_{p}(x, y)=\sum_{i=1}^{N} A_{i} \delta\left(t-\tau_{i}\right) .
$$

where $N$ is the number of eigenrays. 


\subsection{Simulation Result}

For the purpose of simulation, the source position $\left(r_{s}, z_{s}\right)=(1000 m, 20 m)$ and the receiver position $\left(r_{0}, z_{0}\right)=(0 m, 0 m)$ is used. Sound speed profile used to model the channel is shown in Fig. 4(a). Hypothetically, channel modeled from passive source to receiver is equal to actual channel. $S N R$ is defined at output of filter. Location result of one target by DTRM is shown in Fig. 4(b), while $S N R=-4 d B$ and bandwidth from $2.5 \mathrm{kHz}$ to $7.5 \mathrm{kHz}$ over a 0.5 -s time window.

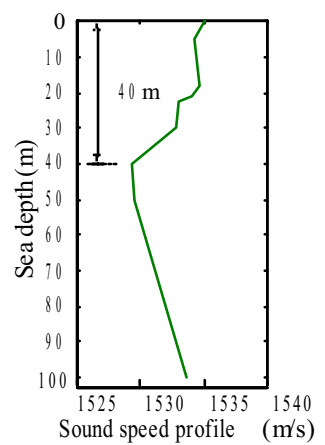

(a) Sound speed profile used to establish the channel model

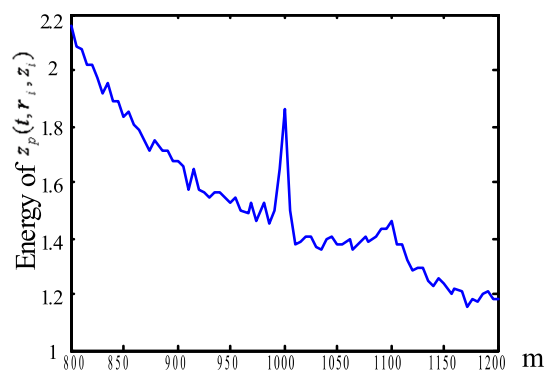

(b) Location result of one target only by DTRM (Range changed

Fig. 4. Simulation result of DTRM

In Fig. 4(b), x-axis denotes range $r_{i}$ of observer and y-axis denotes DTRM's energy output at observer. Simulation result proves the passive location ability of TRM by one sensor.

Three-dimensional location result by DTRM is shown in Fig. 5, in which the source position $\left(r_{s}, z_{s}\right)=(1000 m, 40 m)$ relative to the receiver. Color in the figure shows the energy output of DTRM.

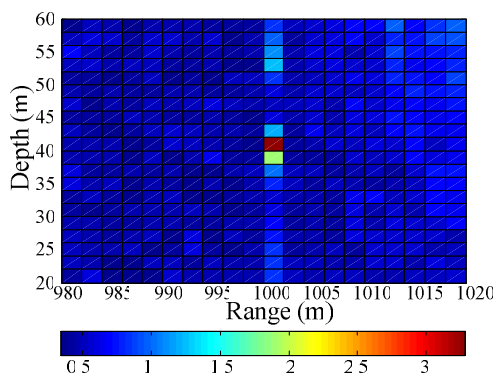

Fig. 5. The DTRM refocused energy distribution in shallow sea 
The peak value of energy output is present at range $1000 \mathrm{~m}$ to $1020 \mathrm{~m}$, depth $40 \mathrm{~m}$ to $42 \mathrm{~m}$, which mean that the source can be located passively by the DTRM refocused energy distribution.

\section{DTRM Experiment}

An experiment conducted in the ocean in 2006 demonstrated that a DTRM can be implemented to locate a passive source. Fig. 6(a). is a schematic of the experiment. The DTRM was implement by one receive sensor in 160-m-deep water. An underwater explosive sound source was used as a passive source which distance from receive sensor was $9.4 \mathrm{~km}$ measured by GPS.

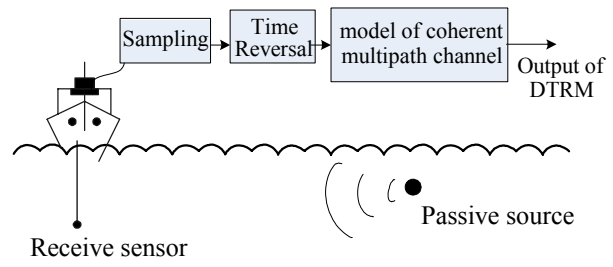

(a) Experimental setup

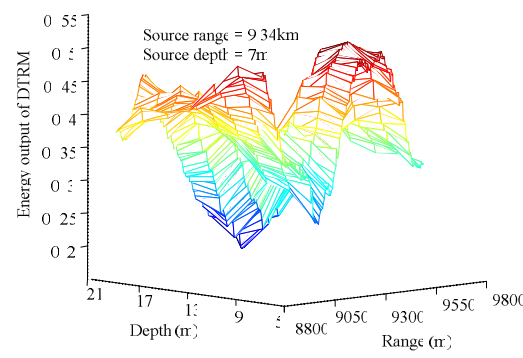

(b) Energy output of DTRM

Fig. 6. DTRM experiment in the ocean

Energy output of DTRM is shown in Fig. 6(b). The waterfall plot shows the energy output of DTRM as a function of depth and range. Maximum energy strength of dummy propagation using data got by receive sensor indicates the source range at 9.3 $\mathrm{km}$ and depth at $7 \mathrm{~m}$, which accord with the result got by GPS. This experiment demonstrated that a DTRM can be implemented to refocus a passive source back to its origin in the ocean.

The second experiment was performed off the Songhua lake of China in September 2007 as indicated in Fig. 7. A three- vector-buoy acoustic positioning array was used

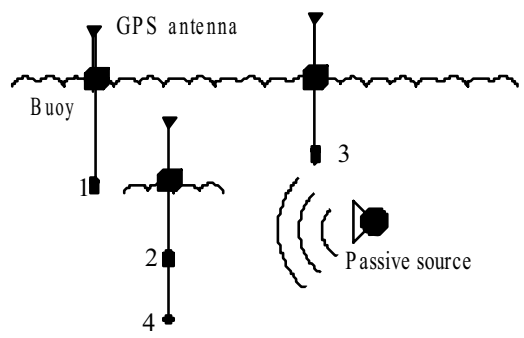

Fig. 7. Experimental setup of DTRM experiment in the lake 


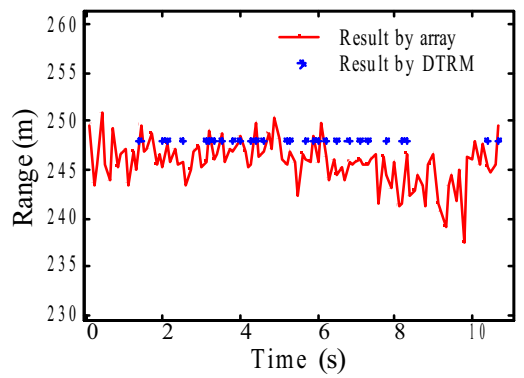

(a) Location of Range

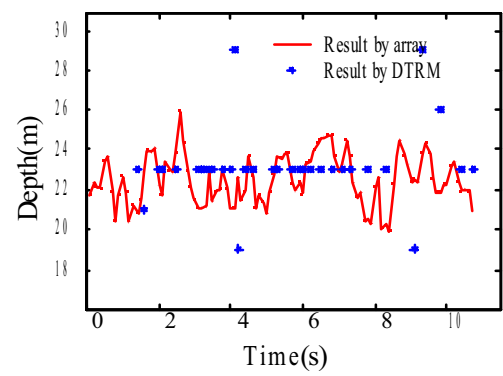

(b) Location of Depth

Fig. 8. Location results of experiment in the lake

to operate passive source's three-dimension location. 1, 2 and 3 indicated in Fig. 7 were vector sensors in 16-m, 8-m and 14-m deep, and 4 was press sensor in 22-m deep. The DTRM was implemented by sensor 2 . The bandwidth of passive source is from $800 \mathrm{~Hz}$ to $2000 \mathrm{~Hz}$.

Locating result obtained by buoy array is shown in Fig. 8.by line and DTRM by dot.

Compared with classically passive location method using buoy array, DTRM also can locate passive source accurately merely several inefficacy refocus appears during the process.

The third experiment in the ocean in April 2006 demonstrated that DTRM with one receiver can be implemented to locate a passive moving source as well.

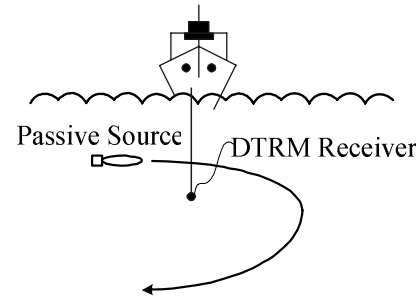

(a) Experimental setup

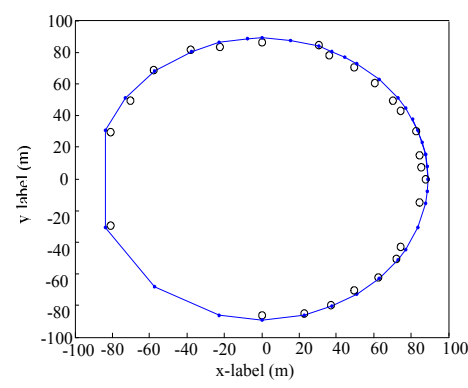

(b) Location results

Fig. 9. Experiment of DTRM to locate a passive moving source

Fig. 9 (a) is a schematic of the experiment. Receiver is 5-elements vector array and DTRM receiver indicated by one of them. Passive source move around the array and two- dimension's location result obtained by using array is shown in Fig. 9 (b) as the lines and DTRM' result as dot. This may be a result that DTRM can be implemented to locate a passive moving source. 


\section{Conclusion}

The concept of the Dummy Time Reversal Mirror is introduced. This is used to locate passive point source in the ocean. We have implemented acoustic DTRM in the ocean and hence demonstrated that DTRM is realizable in ocean using a receive sensor and rather simple signal processing. We also have investigated that DTRM can be implemented to locate a passive moving source as well as it would to motionless source.

\section{References}

1. Fink, M., Prada, C., Wu, F., Cassereau, D.: Self focusing in homogeneous media with 'time reversal' acoustic mirror. In: Proceedings of the IEEE Ultrasonic Symposium 1989, Montreal, PQ, Canada, pp. 681-686 (1989)

2. Jackson, D.R., Dowling, D.R.: Phase Conjugation in underwater acoustic. J. Acoust. Soc. Am. 89, 171-181 (1991)

3. Kuperman, W.A., Hodgkiss, W.S., Song, H.C.: Phase conjugation in the ocean: Experimental demonstration of an acoustic time-reversal mirror. J. Acoust. Soc. Am. 103(1), 2540 (1998)

4. Edelmann, G.F., Akal, T., Kuperman, W.A.: An initial demonstration of underwater acoustic communications using time reversal. IEEE J. Ocean. Eng. 27, 602-609 (2002)

5. Song, H.C., Hodgkiss, W.S., Kuperman, W.A., Sabra, K.G., Akal, T.: Passive reverberation nulling for target enhancement. J. Acoust. Soc. Am. 122(6), 3296-3303 (2007)

6. Jackson, D.R., Dowling, D.R.: Narrow-band performance of phase-conjugate arrays in dynamic random media. J. Acoust. Soc. Am. 91, 3257-3277 (1992)

7. Dowling, D.R.: Phase-conjugate array focusing in a moving medium. J. Acoust. Soc. Am. 94, 1716-1718 (1993)

8. Hui, J., Sheng, X.: Underwater Acoustic Channel, 2nd edn. Defense Industry Press, Beijing (2007)

9. Fan, M.: A Study on Simulation \& Application of Underwater Sound Channel. A Dissertation for the Degree of D.Eng, Harbin Engineering University (2000)

10. Brekhovskikh, L.M.: Fundamentals of Ocean Acoustics. Science Press, Beijing (1983)

11. Wang, D., Shang, E.: Underwater Acoustics. Science Press, Beijing (1981) 